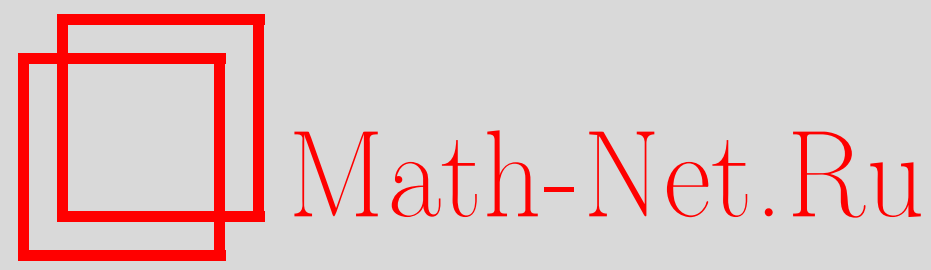

В. А. Скворцов, О теореме Марцинкевича для двоичного интеграла Перрона, Матем. заметки, 1996, том 59, выпуск 2, 267-277

DOI: https://doi.org/10.4213/mzm1713

Использование Общероссийского математического портала MathNet.Ru подразумевает, что вы прочитали и согласны с пользовательским соглашением

http://www. mathnet.ru/rus/agreement

Параметры загрузки:

IP : 54.162 .127 .20

26 апреля 2023 г., $17: 25: 32$

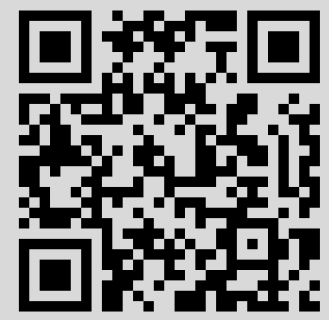




\section{О ТЕОРЕМЕ МАРЦИНКЕВИЧА ДЛЯ ДВОИЧНОГО ИНТЕГРАЛА ПЕРРОНА}

\section{В. А. Скворцов}

Теорема Марцинкевича, о которой идет речь в данной заметке, утверждает, что измеримая функция $f:[a, b] \rightarrow \mathbb{R}$ интегрируема по Перрону тогда и только тогда, когда она обладает по крайней мере одной непрерывной перроновской мажорантой и одной непрерьвной перроновской минорантой (см. [1]). С тех пор, как эта теорема была впервые опубликована в первом издании книги Сакса [1] (см. также [2], где эта теорема была получена независимо) она была обобщена на случай более общих интегралов перроновского типа (см. [3], [4], [5], а также обзорную статью [6]). В то же время было замечено, что для некоторых интегралов, которые определяются с помощью симметричных производных чисел, теорема Марцинкевича неверна (см. [7], [6], [8]).

В этой заметке мы сделаем некоторые общие наблюдения, касающиеся взаимосвязи этой теоремы с понятием вариации функции относительно дифференциального базиса, и затем, на основе этих наблюдений покажем, что теорема Марцинкевича неверна для двоичного интеграла Перрона.

1. Напомним некоторые определения (см. [9], [10]). Дифференииальным.м базисом $\beta$ назовем некоторое подмножество декартова произведения $J \times \mathbb{R}$, где $\mathbb{R}$ - действительная прямая, а $J$ - множество всех невырожденных (замкнутых интервалов) на $\mathbb{R}$.

Если $E \subset \mathbb{R}$, то положим

$$
\begin{gathered}
\beta(E)=\{(I, x) \in \beta ; I \subset E\}, \\
\beta[E]=\{(I, x) \in \beta ; x \in E\} .
\end{gathered}
$$

Скажем, что дифференциальный базис $\beta$ задан на отрезке $I_{0}$, если $\beta=$ $\beta\left(I_{0}\right)=\beta\left[I_{0}\right]$. Множество $\beta[\{x\}]$ будем назьвать дифференииальным $6 а$ зисом в точке $x$.

Работа вьполненапри финансовой поддержке Российского фонда фундаментальных исследований, грант № 94-01-00417. 
Если функция $\delta(x)>0$ определена на множестве $E \subset \mathbb{R}$, положим

$$
\beta_{\delta}[E]=\{(I, x) \in \beta[E]: I \subset(x-\delta(x), x+\delta(x))\}
$$

Все дальнейшие рассмотрения будем вести на некотором фиксированном интервале, на котором задан базис $\beta$. Пусть это будет интервал $[a, b]$. Мы будем предполагать, что для любой функции $\delta(x)>0$, заданной на $[a, b]$, и любого $x \in[a, b]$ вьполнено соотношение $\beta_{\delta}[\{x\}] \neq \varnothing$ (свойство Витали).

Конечное подмножество $\pi \subset \beta_{\delta}[E]$ назовем $\beta_{\delta}$ разбиением на множсестве $E$, если интервалы $I_{1}$ и $I_{2}$ из различных пар $\left(I_{1}, x_{1}\right) \in \pi$, $\left(I_{2}, x_{2}\right) \in \pi$ не перекрьваются. Разбиение $\pi \subset \beta_{\delta}(I) \cap \beta_{\delta}[I]$ назовем $\beta_{\delta}$-разбиением отрезка $I$, если

$$
\bigcup_{i:\left(I_{i}, x_{i}\right) \in \pi} I_{i}=I .
$$

Скажем, что базис $\beta$ обладает свойством разбиения, если для любого интервала $I$ такого, что $(I, x) \in \beta$ при некотором $x$ и для любой функции $\delta(x)>0$, заданной на $[a, b]$, существует $\beta_{\delta}$-разбиение интервала $I$.

Все рассматриваемые здесь функции предполагаются действительно-

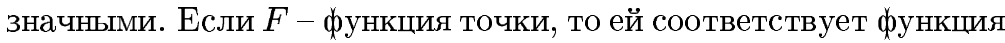

$$
F([a, b])=F(b)-F(a) .
$$

Если функция интервала аддитивна, то соотношение (1) позволяет восстановить (с точностью до аддитивной константы) функцию точки, для которой верно (1). Мы будем ниже также рассматривать функции, заданные на базисе $\beta$, т.е. на множестве пар "интервал-точка".

Верхнюю и нижнюю производную функции интервала $F$ (или соответствующей ей по (1) функции точки, если $F$ аддитивна) в точке $x$ относительно базиса $\beta$ определим соотношениями

$$
\begin{aligned}
& \bar{D}_{\beta} F(x)=\inf _{\delta}\left\{\sup \frac{F(I)}{|I|}:(I, x) \in \beta_{\delta}[\{x\}]\right\}, \\
& \underline{D}_{\beta} F(x)=\sup _{\delta}\left\{\inf \frac{F(I)}{|I|}:(I, x) \in \beta_{\delta}[\{x\}]\right\} \text {. }
\end{aligned}
$$

Если $\bar{D}_{\beta} F(x)=\underline{D}_{\beta} F(x)$, то это обобщение назовем $\beta$-производной функции $F$ в точке $x$, обозначив ее $D_{\beta} F(x)$. Если эта производная конечна в точке $x$, то скажем, что $F$ дифферениируема в $x$ относительно базиса $\beta$, или, короче, $\beta$-дифферениируема.

Функция интервала $F$ называется супераддитивной, если для интервалов $I, I_{1}, I_{2}$ таких, что $I=I_{1} \cup I_{2}$ и интервалы $I_{1}$ и $I_{2}$ не перекрьваются, 
справедливо неравенство $F(I) \geqslant F\left(I_{1}\right)+F\left(I_{2}\right)$. Функция $F$ назьвается субаддитивной, если $-F$ супераддитивна.

Пусть $f$ - функция точки на $[a, b]$ и $\beta$ - дифференпиальньй базис, заданный на $[a, b]$, причем $([a, b], x) \in \beta$ при некотором $x$. Супераддитивная функция интервала $M$ называется $P_{\beta}$-мажсорантой функции $f$, если $\underline{D}_{\beta} M(x) \geqslant f(x)$ для всех $x \in[a, b]$. Субаддитивная функция интервала $m$ назьвается $P_{\beta}$-минорантой функции $f$, если $\bar{D}_{\beta} m(x) \leqslant f(x)$ для всех $x \in[a, b]$. Для базисов, обладающих свойством разбиения, справедливо (см. [9]) соотношение $M(I) \geqslant m(I)$ при любом $I$ таком, что $(I, x) \in \beta$. Поэтому имеет смысл следующее определение: функция $f \quad P_{\beta}$-интегрируема на $[a, b]$ и ее $P_{\beta}$-интеграл равен конечному числу $C$, если $\inf M([a, b])=\sup m([a, b])=C$, где inf и sup берутся по множеству всех $P_{\beta}$-мажорант и по множеству всех $P_{\beta}$-минорант функции $f$ соответственно.

Легко проверить, что если $P_{\beta}$-интеграл функции $f$ существует на $[a, b]$, то он определен и на всяком внутреннем подинтервале $I$ таком, что $(I, x) \in \beta$, являясь аддитивной функцией интервала, которую естественно назвать неопределенным $P_{\beta}$-интегралом функции $f$ и обозначить $P_{\beta}-\int_{I} f=F(I)$. Неопределенньй интеграл $F(I)$ непрерывен относительно базиса $\beta$ в каждой точке $x$, т.е. для любого $\varepsilon>0$ существует функция $\delta(x)>0$ такая, что $|F(I)|<\varepsilon$, если $(I, x) \in \beta_{\delta}[\{x\}]$.

Заметим, что классический интеграл Перрона (см. [1]) определяется с помощью мажорант и минорант, являющихся функциями точки, что соответствует использованию аддитивных мажорант и минорант в вьше приведенном определении. В теореме Марцинкевича также речь идет об аддитивных мажорантах и минорантах.

Для базисов, обладающих свойством разбиения, интеграл Перрона эквивалентен интегралу Хенстока-Курцвейля, определяемому с помощью обобщенных сумм Римана (см. [9], [11]). Нам здесь не понадобится само определение этого интеграла, но некоторые понятия и факты из теории интеграла Хенстока-Курцвейля мы будем использовать (см. [9], [10]).

Пусть $\Phi(I, x)$ - функция, определенная на элементах базиса $\beta$, заданного на отрезке $[a, b]$. В частности, это может быть функция интервала $F(I)$, что соответствует условию $F(I)=\Phi\left(I, x_{1}\right)=\Phi\left(I, x_{2}\right)$ при любых $x_{1}$ и $x_{2}$ таких, что $\left(I, x_{1}\right),\left(I, x_{2}\right) \in \beta$.

Определим для фиксированной функции $\delta(x)>0$, заданной на $[a, b]$, и для фиксированного множества $E \subset[a, b] \delta$-вариаиию функиии $\Phi$ относительно базиса $\beta$ по множеству $E$ на отрезке $A \subset[a, b]$, положив

$$
V\left(\beta_{\delta}, \Phi, E, A\right)=\sup \left\{\sum_{(I, x) \in \pi}|\Phi(I, x)|: \pi \subset \beta_{\delta}[E \cap A] \cap \beta_{\delta}(A)\right\}
$$


Согласимся считать, что в случае $E \cap A=\varnothing \delta$-вариация функции $\Phi$ по $E$ на отрезке $A$ равна нулю. Для функции точки $\delta$-вариацию будем считать совпадающей по определению с $\delta$-вариацией соответствующей функции интервала, определенной равенством (1).

Отметим, что при фиксированных $\delta(x), \Phi, E$ вариация

$$
V_{\delta}(A)=V\left(\beta_{\delta}, \Phi, E, A\right)
$$

рассмотренная как функция интервала $A$, очевидно неотрицательна и супераддитивна.

Далее определим $\beta$-вариацию функиии $\Phi$ по множеству $E$ на отрезке $A$ соотношением

$$
V(\beta, \Phi, E, A)=\inf _{\delta} V\left(\beta_{\delta}, \Phi, E, A\right)
$$

где inf берется по всем функциям $\delta(x)>0$. Очевидно, что

$$
V\left(\beta, \Phi, E_{1}, A\right) \leqslant V\left(\beta, \Phi, E_{2}, A\right), \quad \text { если } E_{1} \subset E_{2} .
$$

Роль $\beta$-вариации в теории интеграла Перрона связана со следующими теоремами, которые являются частньми случаями, соответственно, теорем 1.6.1 и 1.6.4 из [9].

Теорема А. Пусть базис $\beta$ обладает свойством разбиения. Пусть функция $f \quad P_{\beta}$-интегрируема на $[a, b]$ и $G(I)$ - ее неопределенный $P_{\beta}$-интеграл. Тогда для функиии, определенной для каждого әлемента $(I, x)$ базиса $\beta$ равенством $\Phi(I, x)=G(I)-f(x)|I|$, справедливо соотношение

$$
V(\beta, \Phi,[a, b],[a, b])=0
$$

ТЕОРема В. Пусть базис $\beta$ обладает свойством разбиения на $[a, b]$. Пусть $F(I)$ - аддитивная функиия интервала, а $f(x)$ - функиия точ$\kappa u$, заданная на $[a, b]$. Пусть $D=\left\{x: D_{\beta} F(x)=f(x)\right\}$. Тогда $f$ $P_{\beta}$-интегрируема на $[a, b]$ и $F(I)$ - ее неопределенный $P_{\beta}$-интеграл, если

$$
V(\beta, F(I)-f(x)|I|,[a, b] \backslash D,[a, b])=0 .
$$

Из этих теорем нетрудно вывести следующую теорему, которая будет полезным орудием для построения контрпримеров при выяснении возможности обобщения теоремы Марцинкевича на случай некоторых базисов. 
ТЕОРема 1. Пусть базис $\beta$ обладает свойством разбиения. Пусть $G(I)$ - аддитивная функиия интервала, определенная на всех интервалах $I \subset[a, b]$ таких, что $(I, x) \in \beta([a, b])$ и пусть $E$ - множество точек, в которых не существует конечная производная $D_{\beta} G(x)$. Тогда функиия

$$
f(x)= \begin{cases}D_{\beta} G(x), & \text { если } G \text { дифференцируема в } x, \\ 0, & \text { если } x \in E,\end{cases}
$$

$P_{\beta}$-интегрируема на $[a, b]$ и $G(I)$ является ее неопределенным $P_{\beta}$-интегралом тогда и только тогда, когда

$$
V(\beta, G, E,[a, b])=0 .
$$

ДоКАЗАТЕЛЬСТво. Пусть $f P_{\beta}$-интегрируема и $G(I)$ - ее неопределенный $P_{\beta}$-интеграл. Тогда для функции $\Phi$, определенной, как в теореме A, в силу неравенства (5) и утверждения теоремы А имеем

$$
V(\beta, \Phi, E,[a, b])=0 .
$$

Но $f(x)=0$ при $x \in E$. Поэтому $\Phi(I, x)=G(I)$ при $x \in E$ и из (7) следует (6).

Обратное утверждение следует из теоремы В.

Опираясь на теорему 1 , докажем следующее утверждение.

ТЕОРема 2. Пусть базис $\beta$ обладает свойством разбиения на $[a, b]$. Пусть $F(I)$ - аддитивная функиия интервала, определенная на всех интервалах $I \subset[a, b]$ таких, что $(I, x) \in \beta([a, b])$, и $\beta$-дифференцируемая всюду, кроме замкнутого множества Е. Пусть $\left\{\gamma_{n}\right\}-$ множество смехных интервалов к Е. Тогда функиия

$$
f(x)=\left\{\begin{array}{lll}
D_{\beta} F(x), & \text { если } \quad x \in[a, b] \backslash E, \\
0, & \text { если } \quad x \in E
\end{array}\right.
$$

$P_{\beta}$-интегрируема тогда и только тогда, когда найдется аддитивная функиия интервала $H(I)$, для которой $H(I)=0$ при любом I таком, что $(I, x) \in \beta\left(\gamma_{n}\right)$ для некоторого $\gamma_{n}$, и такая, что для функиии

$$
G(I)=F(I)+H(I)
$$

выполняется соотношение

$$
V(\beta, G, E,[a, b])=0 .
$$


ДоКАЗАТЕЛЬСТВо. Поскольку $D_{\beta} G(x)=D_{\beta} F(x)=f(x)$ вне $E$, то к $G$ применима теорема 1 и поэтому из (10) следует $P_{\beta}$-интегрируемость функции $f$.

Для доказательства обратного утверждения отметим, что если функция $f P_{\beta}$-интегрируема, то на любом отрезке $I$ таком, что $(I, x) \in \beta\left(\gamma_{n}\right)$ для некоторого $\gamma_{n}$, ее интеграл совпадает с $F(I)$, так как на $I$ функция $F$ является одновременно мажорантной и минорантной для $D_{\beta} F(x)$. Поэтому неопределенньй $P_{\beta}$-интеграл функции $f$ необходимо имеет представление (9). Остается еще раз применить теорему 1 к функции $G$.

В случаях, когда дается отрицательньй ответ на вопрос о справедливости теоремы Марцинкевича для конкретного базиса $\beta$, теорема 2 позволяет при построении контрпримера доказьвать интегрируемость соответствующей функции. Существование пары мажоранта-миноранта в таком контрпримере также может быть доказано с использованием $\delta$-вариации $F$ из теоремы 2 при условии, что мы требуем аддитивности мажоранты и миноранты. В самом деле, для широкого класса базисов можно показать, что функция интервала $V_{\delta}(I)$, определенная равенством (3) для функции $F$ и множества $E$ из теоремы 2 , является непрерывной супераддитивной функцией интервала (см., например, [8]), и поэтому, если она к тому же конечна на $[a, b]$, функции $F(I)+V_{\delta}(I)$ и $F(I)-V_{\delta}(I)$ образуют, соответственно, супераддитивную непрерывную миноранту и субадлитивную непрерьвную мажоранту функции (8). Однако, во многих случаях, в том числе в рассматриваемом ниже случае двоичного базиса, функция $V_{\delta}(I)$ не является аддитивной (в чем нетрудно убедиться путем построения примера). Поэтому для построения аддитивных непрерьвньх мажорант и минорант мы ниже воспользуемся другим методом.

2. Перейдем непосредственно к рассмотрению случая, ког да $\beta$ - двоичный базис на отрезке $[0,1]$, т.е. базис, образованньй всевозможными парами $(\Delta, x)$, где $x$ - любая точка, принадлежашая $\Delta$, а интервалы $\Delta$ имеют вид

$$
\Delta_{j}^{n}=\left[\frac{j}{2^{n}}, \frac{j+1}{2^{n}}\right], \quad n=0,1,2, \ldots, \quad 0 \leqslant j \leqslant 2^{n}-1 .
$$

Интервалы $\Delta_{j}^{n}$ при фиксированном $n$ назовем интервалами ранга $n$. Двоичный базис очевидно обладает свойством разбиения. Если $x-$ двоично-иррациональная точка, то базис $\beta[\{x\}]$ состоит из элементов $\left(\Delta_{j(x, n)}^{n}, x\right)$, где $\left\{\Delta_{j(x, n)}^{n}\right\}_{n}$ - последовательность вложенных двоичных интервалов, для которых $x$ является общей внутренней точкой. В случае двоично-рациональной точки $x$ базис $\beta[\{x\}]$ образован двумя такими последовательностями интервалов, для которых $x$ является, начиная с некоторого номера $n$, обшим коншом. Интеграл Перрона для двоичного базиса рассмотрен нами в [12]-[14]. 
Покажем, что для двоичного интеграла Перрона несправедлив аналог теоремы Марцинкевича, а имеет место следующее утверждение.

Теорема 3. Существует измеримая на отрезке $[0,1]$ функиия, обладающая непрерывной аддитивной $P_{\beta}$-мажсорантой и непрерывной аддитивной $P_{\beta}$-минорантой, где $\beta$ - двоичный базис, но неинтегрируемая в смысле двоичного интеграла Перрона.

ДокАЗАТЕЛЬСтво. Построим на $[0,1]$ симметричное совершенное множество канторовского типа путем удаления смежных интервалов так, что отношение длины удаляемого открытого интервала к длине концентрического с ним отрезка, из которого он удаляется, постоянно и равно $1 / 2$. Таким образом, на первом шаге мы удаляем из отрезка $[0,1]=\Delta^{0}=\Delta_{0}^{1} \cup \Delta_{1}^{1}$ интервал $(1 / 4,3 / 4)$, оставляя отрезки $\Delta_{0}^{2}$ и $\Delta_{3}^{2}$. Далее построение можно описать по индукции. Пусть после $(n-1)$-го удаления осталось $2^{n-1}$ отрезков, каждый из которых представляет собой двоичньй отрезок $\Delta_{j}^{2 n-2}$ ранга $2 n-2$. Нижние индексы $j$ этих оставшихся двоичных отрезков обозначим $j(k, 2 n-2)$, где $k=0,1, \ldots, 2^{n-1}-1$ и

$$
0=j(0,2 n-2)<j(1,2 n-2)<\cdots<j\left(2^{n-1}-1,2 n-2\right)=2^{2 n-2}-1 .
$$

Каждьй из этих отрезков можно представить в виде

$$
\begin{aligned}
& \Delta_{j(k, 2 n-2)}^{2 n-2}=\Delta_{2 j(k, 2 n-2)}^{2 n-1} \cup \Delta_{2 j(k, 2 n-2)+1}^{2 n-1} \\
& =\Delta_{4 j(k, 2 n-2)}^{2 n} \cup \Delta_{4 j(k, 2 n-2)+1}^{2 n} \cup \Delta_{4 j(k, 2 n-2)+2}^{2 n} \cup \Delta_{4 j(k, 2 n-2)+3}^{2 n} .
\end{aligned}
$$

На $n$-м шаге каждого интервала $\Delta_{j(k, 2 n-2)}^{2 n-2}$ удаляется открытый интервал $\gamma_{k}^{n}$, замыкание которого представимо в виде

$$
\bar{\gamma}_{k}^{n}=\Delta_{4 j(k, 2 n-2)+1}^{2 n} \cup \Delta_{4 j(k, 2 n-2)+2}^{2 n} .
$$

После этого на $\Delta_{j(k, 2 n-2)}^{2 n-2}$ остаются два интервала ранга $2 n: \Delta_{4 j(k, 2 n-2)}^{2 n}$ и $\Delta_{4 j(k, 2 n-2)+2}^{2 n}$. В результате этого построения определяется целочисленная функция $j(k, m)$ для $m=0,1, \ldots$, и для $k$ таких, что $0 \leqslant k \leqslant 2^{n}-1$ при $m=2 n$ и $m=2 n-1$. Значения функции удовлетворяют условию $0 \leqslant j(k, m) \leqslant 2^{m}-1$ и определяются по индукции соотношениями

$$
\begin{aligned}
j(2 k, 2 n-1) & =2 j(k, 2 n-2), & j(2 k+1,2 n-1) & =2 j(k, 2 n-2)+1, \\
j(2 k, 2 n) & =4 j(k, 2 n-2), & j(2 k+1,2 n) & =4 j(k, 2 n-2)+3 .
\end{aligned}
$$

При этом

$$
\begin{aligned}
\Delta_{j(2 k, 2 n)}^{2 n} & \subset \Delta_{j(2 k, 2 n-1)}^{2 n-1} \subset \Delta_{j(k, 2 n-2)}^{2 n-2}, \\
\Delta_{j(2 k+1,2 n)}^{2 n} & \subset \Delta_{j(2 k+1,2 n-1)}^{2 n-1} \subset \Delta_{j(k, 2 n-2)}^{2 n-2}
\end{aligned}
$$


Отметим (см. [11], [12], [13]), что при любых $k$ и $n$

$$
\Delta_{j(k, 2 n-1)}^{2 n-1} \backslash \Delta_{j(k, 2 n)}^{2 n} \subset \gamma_{k}^{n} .
$$

Положим

$$
E_{n}=\bigcup_{k=0}^{2^{n}-1} \Delta_{j(k, 2 n)}^{2 n}, \quad E=\bigcap_{n=1}^{\infty} E_{n} .
$$

Очевидно, что $|E|=0$ и что любой точке $x \in E$ соответствует последовательность индексов $\{k(x, m)\}_{m}$ такая, что последовательность интервалов $\left\{\Delta_{j(k(x, m), m)}^{m}\right\}_{m}$ стягивается к точке $x$. Отметим, что у интервалов четного ранга $\Delta_{j(k, 2 n)}^{2 n}$ оба конца принадлежат $E$, в то время как у интервалов $\Delta_{j(k, 2 n-1)}^{2 n-1}$ один конец лежит в $E$, а другой является центром смежного интервала $\gamma_{k}^{n}$.

Пусть $c_{k}^{n}$ - центр интервала $\gamma_{k}^{n}=\left(a_{k}^{n}, b_{k}^{n}\right)$ так, что

$$
\begin{aligned}
& {\left[a_{k}^{n}, c_{k}^{n}\right]=\Delta_{j(2 k, 2 n)+1}^{2 n} \subset \Delta_{j(2 k, 2 n-1)}^{2 n-1},} \\
& {\left[c_{k}^{n}, b_{k}^{n}\right]=\Delta_{j(2 k+1,2 n)-1}^{2 n} \subset \Delta_{j(2 k+1,2 n-1)}^{2 n-1} .}
\end{aligned}
$$

Определим на $[0,1]$ функцию $F(x)=0$, если $x \in E$, и

$$
F(x)=2^{-n} \sin ^{2}\left(\frac{x-a_{k}^{n}}{b_{k}^{n}-a_{k}^{n}} \pi\right), \quad \text { если } x \in\left(a_{k}^{n}, b_{k}^{n}\right)=\gamma_{k}^{n} .
$$

Очевидно, что $F$ непрерывна на всем отрезке [0, 1$]$ и дифференцируема на $[0,1] \backslash E$, причем в коншевых точках $a_{k}^{n}$ и $b_{k}^{n}$ интервала $\gamma_{k}^{n}$ она имеет одностороннюю производную изнутри интервала, равную нулю. Из определения (16) видно, что $F\left(c_{k}^{n}\right)=2^{-n}$ и $F\left(a_{k}^{n}\right)=F\left(b_{k}^{n}\right)=0$, откуда с учетом (15) для соответствующей, согласно (1), функции интервала имеem

$$
\left|F\left(\Delta_{j(k, 2 n-1)}^{2 n-1}\right)\right|=2^{-n} .
$$

Кроме того.

$$
F\left(\Delta_{j(k, 2 n)}^{2 n}\right)=0
$$

Докажем, что функция

$$
f(x)= \begin{cases}F^{\prime}(x), & \text { если } x \in[0,1] \backslash E, \\ 0, & \text { если } x \in E,\end{cases}
$$

обладает аддитивной непрерывной $P_{\beta}$-мажорантой и аддитивной непрерывной $P_{\beta}$-минорантой. Мажоранту и миноранту мы определим как функции интервала, равные прирашениям соответствуюших функций точки, чем будет гарантирована их аддитивность. 
Определим на множестве $E$ сингулярную меру $\mu$ так, чтобы для каждого интервала $\Delta_{j(k, 2 n)}^{2 n}$ выполнялось равенство

$$
\mu\left(E \cap \Delta_{j(k, 2 n)}^{2 n}\right)=2^{-n} .
$$

Тем самьм

$$
\mu(E)=1 .
$$

Поскольку $E \cap \Delta_{j(k, 2 n-1)}^{2 n-1}=E \cap \Delta_{j(k, 2 n)}^{2 n}$, то

$$
\mu\left(E \cap \Delta_{j(k, 2 n-1)}^{2 n-1}\right)=\mu\left(E \cap \Delta_{j(k, 2 n)}^{2 n}\right)=2^{-n} .
$$

Определим функцию точки на $[0,1]$, положив $r(x)=\mu(E \cap[0, x])$. Эта функция непрерывна и монотонна на $[0,1]$ и постоянна на каждом интервале $\gamma_{k}^{n}$. Пусть $R$ - функция интервала, определяемая как прирашение функции $r$. Ясно, что

$$
D_{\beta} R(x)=R^{\prime}(x)=0, \quad \text { если } x \in[0,1] \backslash E .
$$

Докажем, что в каждой точке $x \in E$ для любого интервала $\Delta_{j}^{m}$ такого, что $x \in \Delta_{j}^{m}$, вьполняется неравенство

$$
\left|F\left(\Delta_{j}^{m}\right)\right| \leqslant R\left(\Delta_{j}^{m}\right) .
$$

В самом деле, если $x \in \Delta_{j}^{m}$, то это значит, что этот интервал имеет вид либо $\Delta_{j(k, 2 n)}^{2 n}$, либо $\Delta_{j(k, 2 n-1)}^{2 n-1}$. В первом случае (22) очевидно в силу (18). Во втором случае имеет место равенство (17), и поэтому в силу (20) и определения функции $R$ имеем

$$
\left|F\left(\Delta_{j(k, 2 n-1)}^{2 n-1}\right)\right| 2^{-n}=\mu\left(E \cap \Delta_{j(k, 2 n-1)}^{2 n-1}\right)=R\left(\Delta_{j(k, 2 n-1)}^{2 n-1}\right),
$$

что также обеспечивает (22). Из неравенства (22), дифференцируемости функции $F$ вне $E$, равенства (21) и непрерывности функций $F$ и $r$ легко следует, что функции $M(\Delta)=F(\Delta)+R(\Delta)$ и $m(\Delta)=F(\Delta)-R(\Delta)$ являются, соответственно, непрерывной аддитивной $P_{\beta}$-мажорантой и непрерьвной аддитивной $P_{\beta}$-минорантой функции $f$.

Осталось доказать, что функция $f$ не является $P_{\beta}$-интегрируемой на $[0,1]$. Для этого воспользуемся теоремой 2.

Пусть $H$ - любая функция интервала, равная нулю на любом двоичном интервале, целиком лежашем внутри какого-нибудь интервала $\gamma_{n}^{k}$. Пусть $G(\Delta)=F(\Delta)+H(\Delta)$. В силу $(15),(17)$ и $(18)$

$$
\begin{aligned}
\left|G\left(\Delta_{j(k, 2 n-1)}^{2 n-1}\right)-G\left(\Delta_{j(k, 2 n)}^{2 n}\right)\right| & =\left|G\left(\Delta_{j(k, 2 n-1)}^{2 n-1} \backslash \Delta_{j(k, 2 n)}^{2 n}\right)\right| \\
& =\left|F\left(\Delta_{j(k, 2 n-1)}^{2 n-1} \backslash \Delta_{j(k, 2 n)}^{2 n}\right)\right|=2^{-n} .
\end{aligned}
$$




\section{Поэтому}

$$
\text { либо }\left|G\left(\Delta_{j(k, 2 n-1)}^{2 n-1}\right)\right| \geqslant 2^{-n-1}, \quad \text { либо }\left|G\left(\Delta_{j(k, 2 n)}^{2 n}\right)\right| \geqslant 2^{-n-1} \text {, }
$$

где $\Delta_{j(k, 2 n)}^{2 n} \subset \Delta_{j(k, 2 n-1)}^{2 n-1}$.

Вновь воспользуемся введенной вьше сингулярной мерой $\mu$.

Зафиксируем произвольную функцию $\delta(x)>0$. Рассмотрим на множестве $E$ произвольное $\beta_{\delta}$-разбиение $\pi=\left\{\left(\Delta_{j}, x\right)\right\} \subset \beta_{\delta}[E]$ такое, что

$$
E \subset \bigcup_{j} \Delta_{j}
$$

Мы перейдем от $\pi$ к другому разбиению $\pi^{\prime}=\left\{\left(\Delta_{i}^{\prime}, x_{i}\right)\right\} \in \beta_{\delta}[E]$ с теми же точками $x_{i}$ и с $\Delta_{i}^{\prime} \subset \Delta_{i}$.

Зафиксируем двоичньй интервал $\Delta_{i}$ из $\pi$. Он имеет либо четньй, либо нечетньй ранг. Если $\Delta_{i}=\Delta_{j(k, 2 n-1)}^{2 n-1}$ при некотором $n$, то к нему применимы соотношения (23). Мы включим в $\pi^{\prime}$ в качестве $\Delta_{i}^{\prime}$ тот из интервалов $\Delta_{j(k, 2 n-1)}^{2 n-1}$ и $\Delta_{j(k, 2 n)}^{2 n}$, для которого реализуется неравенство из $(23)$. В любом случае получим с учетом (20)

$$
\left|G\left(\Delta_{i}^{\prime}\right)\right| \geqslant 2^{-n-1}=2^{-1} \mu\left(E \cap \Delta_{i}\right) .
$$

Если же $\Delta_{i}=\Delta_{j(k, 2 m)}^{2 m}$, то в силу соотношений $(14)$, примененных к $n=m+1$, точка $x_{i}$ лежит либо в $\Delta_{j(2 k, 2 m+1)}^{2 m+1}$, либо в $\Delta_{j(2 k+1,2 m+1)}^{2 m+1}$. В любом из этих случаев к соответствующему интервалу нечетного ранга $2 m+1$ применимо только что проведенное выше рассуждение с заменой в $(23) n$ на $m+1$. Это приводит к выбору в качестве $\Delta_{i}^{\prime}$ такого интервала, для которого

$$
\left|G\left(\Delta_{i}^{\prime}\right)\right| \geqslant 2^{-m-2}=2^{-2} \mu\left(E \cap \Delta_{i}\right) .
$$

Итак, при любом $i$ мы перешли от интервала $\Delta_{i}$ к интервалу $\Delta_{i}^{\prime}$, для которого выполняется по крайней мере (25). Суммируя неравенство (25) по $i$ и учитывая (24) и (19), приходим к соотношению

$$
\sum_{i:\left(\Delta_{i}^{\prime}, x_{i}\right) \in \pi^{\prime}}\left|G\left(\Delta_{i}^{\prime}\right)\right| \geqslant 2^{-2} \mu(E)=2^{-2} .
$$

Поскольку функция $\delta(x)>0$ была взята произвольно, то в соответствии с определениями (2) и (4) получаем

$$
V(\beta, G, E,[0,1]) \geqslant 2^{-2} .
$$

Применяя теорему 2 , приходим к выводу, что $f$ не интегрируема в смысле двоичного интеграла Перрона, что завершает доказательство теоремы 3.

Отметим в заключение, что построенная при доказательстве теоремы 3 функция $F$, очевидно является $A C G$-функцией (см. [1]), которая, к тому же, дифференцируема почти всюду. Поэтому $F$ является неопределенньм интегралом Данжуа-Хинчина (см. [15]) своей производной $F^{\prime}(x)=f(x)$. Тем самьм мы установили справедливость следующего утверждения. 
Теорема 4. Существует функиия, которая интегрируема на отрезке $[0,1]$ в смысле интеграла Данжуа-Хинчина, но не интегрируема на $[0,1]$ в смысле двоичного интеграла Перрона.

Московский государственный университет Поступило им. М. В. Ломоносова

\section{СПИСОК ЦИТИРОВАННОЙ ЛИТЕРАТУРЫ}

[1] Сакс С. Теория интеграла. М.: ИЛ, 1949.

[2] Толстов Г. П. Об интеграле Perron’a // Матем. сб. 1939. Т. 5. № 47. C. $647-660$.

[3] Скворцов В.А. Некоторые свойства $C P$-интеграла // Матем. сб. 1963. T. 60 (102). № 3. C. 304-324.

[4] Bullen P.S. The Burkill approximately continuous integral -II // Math. Chron. 1983. V. 12. P. 93-98.

[5] Байгожин Е.С. О двоичном интеграле Перрона // Вестн. МГУ. Сер. 1. Матем., мех. 1993. № 4.

[6] Bullen P.S. Some applications of a theorem of Marcinkiewicz // Lecture Notes in Math. V. 1419. Berlin: Springer, 1990. P. 10-18.

[7] Скляренко В. А. Обобщенные интегралы в теории тригонометрических рядов. Дисс. ... к.ф.-м.н. М.: МГУ, 1973.

[8] Skvortsov V.A., Thomson B.S. Symmetric integrals do not have the Marcinkiewicz property // Real Analysis Exchange. 1995. V. 20. № 2.

[9] Ostaszewski K. M. Henstock integration in the plane // Amer. Math. Soc. Memoirs. 1986. V. 63. № 353 .

[10] Thomson B.S. Derivates of interval functions // Amer. Math. Soc. Memoirs. 1991. V. 93. № 452.

[11] Henstock R. The general theory of integration. Oxford: Clarendon Press, 1991.

[12] Скворцов В.А. О рядах Хаара, сходящихся по подпоследовательностям частичных сумм // Докл. АН СССР. 1968. Т. 183. № 4. С. 784-786.

[13] Скворцов В. А. Некоторое обобщение интеграла Перрона // Вестн. МГУ. Cер. 1. Матем., мех. 1969. № 4. С. 48-51.

[14] Skvortsov V. A. Some properties of dyadic primitives // Lecture Notes in Math. V. 1419. Berlin: Springer, 1990. P. 167-179.

[15] Хинчин А.Я. О процессе интегрирования Данжуа // Матем. сб. 1918. T. 30. № 4. C. 548-557. 